\section{AB0288 GLUCOCORTICOID SPARING EFFECT OF THE BIOLOGIC DISEASE MODIFYING ANTI-RHEUMATIC DRUGS IN RHEUMATOID ARTHRITIS IN TUNISIAN REAL LIFE PRACTICE}

R. Fakhfakh ${ }^{1}$, H. Zeglaoui ${ }^{1}$, N. El Amri ${ }^{1}$, S. Hamila ${ }^{1}$, N. Amara ${ }^{1}$, K. Baccouche ${ }^{1}$, E. Bouajina ${ }^{1}$ on behalf of BINAR. ${ }^{1}$ Farhat Hached Hospital, Rheumatology, Sousse, Tunisia

Background: Glucocorticoids (GCs) are still widely prescribed in rheumatoid arthritis (RA). Despite their disease-modifying properties, they are associated with significant adverse effects. The international guidelines recommend the lowest effective dose and the lowest duration of GCs. Previous studies have shown that biologic disease modifying anti-rheumatic drugs (bDMARDs) can have a GC-sparing effect in RA.

Objectives: The aim of the study was to assess the impact of the bDMARDs on glucocorticoids use in rheumatoid arthritis Tunisian patients, in real life practice. Methods: RA patients (according to the American College of Rheumatology criteria) who started their first bDMARDs (Tumour necrosis factor(TNF)a inhibitors, Tocilizumab and Rituximab) between January 2016 and august 2019, were recruited from the BINAR « Biologic National Registry", a prospective national Tunisian biologic registry. Oral prednisone intakewascompared at inclusion (M0), at 3 months (M3)and at 6 months(M6) after bDMARDs initiation.

Results: 175 patients were included (149 females / 26 males). The mean age was 54.1 years \pm 12.6 and the mean disease duration was 6.7 years \pm 3.5. The TNFainhibitors, the Tocilizumab and the Rituximab were prescribed, respectively, in $79.4 \%, 17.7 \%$ and $8.6 \%$. The mean DAS28 index activity was $4.9 \pm 1.5$ at M0, $4.5 \pm 1.5$ at M3 and $4.2 \pm 1.1$ at M6 $(\mathrm{p}=0.78)$. At inclusion, 150 patients $(85.7 \%)$ were taking oral prednisone and the mean dose was $8.2 \pm$ $5.4 \mathrm{mg} /$ day. Overall, a significant decrease of oral prednisone use was observed at $\mathrm{M} 3(78 \%)$ and $\mathrm{M} 6(67.6 \%)$. The mean daily dose of oral prednisone was $8.9 \pm 4.3 \mathrm{mg}$ at $\mathrm{M} 3(\mathrm{p}>0.05)$ and $8.1 \pm 2.7 \mathrm{mg}$ at $\mathrm{M} 6(\mathrm{P}>0.05)$. At M3 and M6, $4 \%$ and $2 \%$ of patients $(p>0.05)$ had lowered prednisone doses, respectively. Prednisone discontinuation was observed in $17.7 \%$ at $\mathrm{M} 3$ and $18.1 \%$ at $\mathrm{M} 6$. Increased prednisone doses were noted in $2.7 \%$ at M3 and $2.6 \%$ at M6. The bDMARDs use wasn't associated with oral prednisone decrease at M3 (TNFi $\mathrm{p}=0.51$; Tocilizumab $\mathrm{p}=0.54$; Rituximab $\mathrm{p}=0,77$ ) and at M6 (TNFi $\mathrm{p}=0.61$; Tocilizumab $\mathrm{p}=0.39$; Rituximab $\mathrm{p}=0,64$ ).

Conclusion: This study showed a small glucocorticoids sparing-effect of bDMARDs at 3 months and 6 months in rheumatoid arthritis patients with a decrease of oral prednisone use of $18.1 \%$ at 6 months.

References:

[1] Fortunet c, Pers YP, Lambert $\mathrm{J}$ et al. Tocilizumab induces corticosteroid sparing in rheumatoid arthritis patients in clinical practice. Rheumatology 2015;54:672_677 doi:10.1093/rheumatology/keu339

Disclosure of Interests: None declared

DOI: 10.1136/annrheumdis-2020-eular.6148

\section{AB0289 EFFICACY, SAFETY AND IMMUNOGENICITY IN PATIENTS WITH RHEUMATOID ARTHRITIS COMPARING PF-06410293 (ADL-PF), AN ADALIMUMAB (ADL) BIOSIMILAR, AND REFERENCE ADL: RESULTS FROM WEEK 26-52 OF A DOUBLE-BLIND, RANDOMISED PHASE 3 STUDY INCLUDING PATIENTS WHO SWITCHED FROM ADL-PF TO REFERENCE ADL AT WEEK 26}

R. Fleischmann ${ }^{1}$, D. Alvarez ${ }^{2}$, A. Bock ${ }^{3}$, C. Cronenberger ${ }^{2}$, I. Vranic ${ }^{4}$,

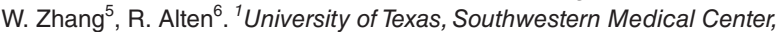
Metroplex Clinical Research Center, Dallas, United States of America; ${ }^{2}$ Pfizer Inc, Collegeville, United States of America; ${ }^{3}$ Pfizer Inc, Cambridge, United States of America; ${ }^{4}$ Pfizer, Tadworth, United Kingdom; ${ }^{5}$ Pfizer Inc, Lake Forest, United States of America; ${ }^{6}$ Schlosspark Klinik, University Medicine, Berlin, Germany

Background: PF-06410293 (ADL-PF) is an adalimumab biosimilar approved for the treatment of several inflammatory and autoimmune indications. ${ }^{1}$ The efficacy, safety and immunogenicity of ADL-PF and reference adalimumab sourced from the European Union (ADL-EU) in patients with rheumatoid arthritis (RA) have been demonstrated to be similar in a randomised controlled trial up to 26 weeks (wks; treatment period 1 [TP1]). ${ }^{2}$

Objectives: To evaluate the efficacy, safety and immunogenicity of ADL-PF and $A D L-E U$ in patients with moderate to severe RA on longer-term treatment, and following a treatment switch from ADL-EU to ADL-PF in a subset of patients. Methods: This multinational, randomised, double-blind, parallel-group study compared ADL-PF and ADL-EU in essentially biologic-naïve patients with active RA despite methotrexate (MTX) (NCT02480153). In TP1, patients were randomised (1:1) to ADL-PF or ADL-EU (40 mg subcutaneous injection every 2 wks) for 26 wks while continuing MTX (10-25 mg/wk). The primary endpoint was achievement of American College of Rheumatology response (ACR20) at Wk 12. At Wk 26, the start of treatment period 2 (TP2), patients receiving ADL-EU were blindly re-randomised (1:1) to remain on ADL-EU or switch to ADL-PF for 26 wks while patients receiving ADL-PF continued treatment in a blinded manner. Secondary efficacy endpoints at Wks 26, 30, 36, 44 and 52 (ACR20/50/70, European League Against Rheumatism [EULAR] response, Disease Activity Score [DAS] 28-4[CRP] $<2.6$ and ACR/EULAR defined remission), safety events and percentage of patients with anti-drug antibodies (ADA) were assessed.

Results: In TP1, 597 patients were randomised to ADL-PF ( $\mathrm{n}=297)$ or ADL-EU $(n=300)$. At Wk 26, 552 patients were re-randomised for TP2 (continued ADL-PF $n=283$; continued ADL-EU, $n=135$; switched from ADL-EU to ADL-PF, $n=134$ ). Patients who demonstrated at least minimal efficacy continued in TP2. Observed ACR20 rates were comparable between treatment groups at all visits during TP2 (Figure). Other measures of deep response (ACR70, EULAR good response, DAS28-4(CRP) <2.6 and ACR/EULAR defined remission) showed maintained efficacy during TP2 in all treatment groups. Incidences of treatment-emergent adverse events were comparable between treatment groups (Table). Overall, incidences of ADA through Wk 52 were comparable between treatment groups (47.3\%, $54.1 \%$ and $45.9 \%$ for patients who continued ADL-PF, continued ADL-EU or switched from ADL-EU to ADL-PF, respectively). In patients who switched from ADL-EU to ADL-PF compared with patients who continued ADL-EU, the increase in ADA incidence over TP2 was $0.8 \%$ (from $45.1 \%$ to $45.9 \%$ ) versus $6.7 \%$ (from $47.4 \%$ to $54.1 \%$ ), respectively.

Conclusion: TP2 results demonstrated comparable efficacy, safety and immunogenicity between ADL-PF and ADL-EU was maintained up to Wk 56 and was unaffected by a blinded switch from ADL-EU to ADL-PF at Wk 26.

References:

[1] Pfizer Inc, 2019. http://labeling.pfizer.com/ShowLabeling.aspx?id=12780

[2] Fleischmann RM et al, Arthritis Res Ther 2018;20:178.

Figure ACR20 response rates by visit: Treatment Period 2 (ITT population)

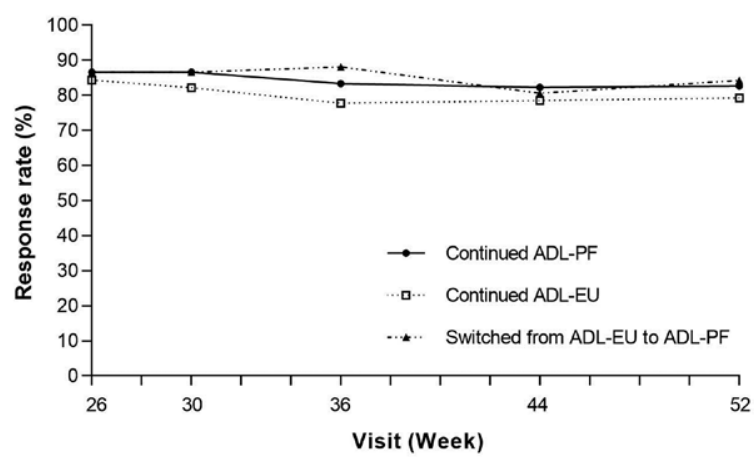

ACR20, $\geq 20 \%$ improvement in American College of Rheumatology response ADL-EU, adalimumab sourced from the European Union; ADL-PF, adalimuma biosimilar PF-06410293; ITT, intent-to-treat.

Table. All-causality TEAEs: Treatment Period 2 (Safety population)

\begin{tabular}{lccc}
\hline & $\begin{array}{c}\text { Continued } \\
\text { ADL-PF } \\
(n=283)\end{array}$ & $\begin{array}{c}\text { Continued } \\
\text { ADL-EU } \\
(n=135)\end{array}$ & $\begin{array}{c}\text { Switched from } \\
\text { ADL-EU to } \\
\text { ADL-PF } \\
(n=133)\end{array}$ \\
\hline Number of AEs & 243 & 112 & 100 \\
Patients with events, $n(\%)$ & $123(43.5)$ & $60(44.4)$ & $51(38.3)$ \\
$\quad$ AEs & $4(1.4)$ & $6(4.4)$ & $3(2.3)$ \\
Serious AEs & $7(2.5)$ & $7(5.2)$ & $4(3.0)$ \\
$\geq$ Grade 3 AEs & $6(2.1)$ & $8(5.9)$ & $2(1.5)$ \\
TEAEs leading to treatment & 0 & 0 & 0 \\
discontinuation & 0 & 0 & \\
Deaths & & &
\end{tabular}

ADL-EU, adalimumab sourced from the European Union; ADL-PF, adalimumab biosimila PF-06410293; AE, adverse event; TEAE, treatment-emergent AE.

Acknowledgments: Medical writing support, provided by Jacqui Oliver of Engage Scientific Solutions. The study was funded by Pfizer.

Disclosure of Interests: Roy Fleischmann Grant/research support from: AbbVie, Akros, Amgen, AstraZeneca, Bristol-Myers Squibb, Boehringer, Ingelh Centrexion, Eli Lilly, EMD Serono, Genentech, Gilead, Janssen, Merck, Nektar, Novartis, Pfizer, Regeneron Pharmaceuticals, Inc., Roche, Samsung, Sandoz, Sanofi Genzyme, Selecta, Taiho, UCB, Consultant of: AbbVie, ACEA, Amgen, Bristol-Myers Squibb, Eli Lilly, Gilead, GlaxoSmithKline, Novartis, Pfizer, Sanof 